\title{
Recrystallization-induced Precipitation Interaction in a Medium Carbon Vanadium Microalloyed Steel
}

\author{
A. QUISPE, S. F. MEDINA and P. VALLES ${ }^{1)}$ \\ Centro Nacional de Investigaciones Metalúrgicas (CENIM-CSIC), Av. Gregorio del Amo, 8; 28040, Madrid, Spain. \\ 1) Instituto Nacional de Técnica Aeroespacial (INTA), Torrejón de Ardoz, Madrid, Spain.
}

(Received on December 24, 1996; accepted in final form on April 11, 1997)

\begin{abstract}
Using torsion tests, a study has been made of Recrystallization-Precipitation (R-P) interaction in a vanadium microalloyed steel for two strains $(0.20$ and 0.35$)$. When strain induced precipitation starts, the recrystallized fraction deviates from Avrami's equation, giving rise to the formation of a plateau on the curves which represent the recrystallized fraction against time. This makes it possible to know the moment at which precipitation starts $\left(P_{\mathrm{s}}\right)$ and the moment at which it finishes $\left(P_{\mathrm{f}}\right)$. After the end of precipitation, recrystallization continues to progress in accordance with Avrami's law. Recrystallization-PrecipitationTime-Temperature (RPTT) diagrams have been drawn, superposing on them the lines corresponding to different recrystallized fractions. This makes it possible to represent in graph form the RecrystallizationPrecipitation interaction and the determination of the static recrystallization critical temperature (SRCT). It is demonstrated that during the interval of time in which precipitation occurs $\left(P_{\mathrm{f}}-P_{\mathrm{s}}\right)$, recrystallization does not advance. The work which has been carried out establishes new aspects in the phenomenon of R-P interaction.
\end{abstract}

KEY WORDS: static recrystallization; precipitation; RPTT diagrams.

\section{Introduction}

After undergoing hot deformation, the static recrystallization of microalloyed steels is clearly divided into two separate stages: before and after the start of induced precipitation. In the first stage the recrystallized fraction against time curves are similar to those for non microalloyed steels and in the second stage the curves display a plateau, caused by the formation of precipitates which inhibit recrystallization. ${ }^{1-4)}$ Before and after the plateau, the recrystallized fraction curve obeys Avrami's law and this makes it possible to easily draw precipitation-timetemperature (PTT) diagrams. ${ }^{2-4)}$ The limit between the two stages comes at a point which will henceforth be described as the Static Recrystallization Critical Temperature (SRCT), or the temperature at which the inhibition of static recrystallization begins as a consequence of induced precipitation.

The vanadium content hardly influences static recrystallization kinetics when the elements are in solution, but has a notable influence on the incubation time of the precipitates and on the value of SRCT. ${ }^{5}$

Recently, a method has been developed to simultaneously determine static recrystallization kinetics and PTT diagrams for microalloyed steels. ${ }^{2-7)}$ This method has been employed in the present work. Analysis of the results has suggested the suitability of simplifying it and thus improving it, providing new ideas about $\mathrm{R}-\mathrm{P}$ interaction. The PTT diagrams for microalloyed steels, published by other authors, were determined by the use of theoretical equations, ${ }^{8-12)}$ transmission microscopy ${ }^{13)}$ and the stress relaxation method. ${ }^{14,15)}$

This work thus presents a study of static recrystallization kinetics, measured at different temperatures, and of induced precipitation kinetics in a medium carbon vanadium microalloyed steel. The $\mathrm{R}-\mathrm{P}$ interaction for different recrystallized fraction percentages has made it possible to determine SRCT with precision and simplicity.

\section{Experimental Procedure}

The steel used was manufactured by Electroslag Remelting (ESR) in a laboratory unit capable of producing $30 \mathrm{~kg}$ ingots and its composition is shown in Table 1. This technique avoids macrosegregation, both in alloying elements and impurities, and there is considerably less microsegregation, these defects being present in conventional ingots and continuous casting billets. The torsion specimens, with a useful length of 50 and $6 \mathrm{~mm}$ in diameter, were austenitized at $1200^{\circ} \mathrm{C}$ for $10 \mathrm{~min}$ and then the temperature was rapidly reduced to the testing temperature and maintained at that temperature for a

Table 1. Chemical composition (wt \%) and austenite grain size $\left(D_{\gamma}\right)$ at $1200^{\circ} \mathrm{C} \times 10 \mathrm{~min}$ in the steel studied.

\begin{tabular}{ccccccccc}
\hline $\mathrm{C}$ & $\mathrm{Si}$ & $\mathrm{Mn}$ & $\mathrm{V}$ & $\mathrm{S}$ & $\mathrm{P}$ & $\mathrm{N}$ & $\mathrm{Al}$ & $\mathrm{D}_{\gamma}(\mu \mathrm{m})$ \\
\hline 0.37 & 0.24 & 1.42 & 0.120 & 0.013 & 0.011 & 0.0151 & 0.012 & 157 \\
\hline
\end{tabular}


time of no more than $60 \mathrm{~s}$.

The magnitudes of torsion (torque, No. of revolutions) and the equivalent magnitudes (stress, strain) have been related in accordance with Von Mises criterion. ${ }^{16)}$ The recrystallized fraction was determined at different temperatures, at the equivalent strains of 0.20 and 0.35 , at a constant strain rate of $3.63 \mathrm{~s}^{-1}(=1000 \mathrm{rev} / \mathrm{min})$.

The recrystallized fraction has been measured using the method known as back extrapolation. ${ }^{17)}$ It has been found that using this method the softened fraction and the recrystallized fraction are approximately equal. ${ }^{18)}$

\section{Results and Discussion}

First of all, the austenite grain size was determined at $1200^{\circ} \mathrm{C} \times 10 \mathrm{~min}$ by means of quenching and subsequent metallographic analysis of 20 fields, applying the linear intersection technique. The average austenite grain size determined was $157 \mu \mathrm{m}$ (Table 1), a value slightly lower than other vanadium steels due to the fact that the high vanadium content is not significant in reducing the austenite grain size. ${ }^{5)}$

Figure 1 shows the recrystallized fraction against time at different temperatures, for a strain of 0.20 and strain rate of $3.63 \mathrm{~s}^{-1}$. The formation of a plateau can be seen on the curves corresponding to 925 and $900^{\circ} \mathrm{C}$, respectively. The plateau is caused by precipitation and its start and finish have been identified with the start and finish of induced precipitation for practical purposes, since before and after the plateau the curve shows kinetics similar to curves corresponding to higher temperatures which do not show a plateau. The plateau appears even in microalloyed steels with very small contents of microalloy in solution, of the order of $0.004 \% \mathrm{wt}^{2)}$ Therefore, if this occurs at very low microalloy contents, it is to be supposed that the start of the plateau coincides approximately with the start of precipitation. At temperatures below $925^{\circ} \mathrm{C}$ the curves always show the formation of the plateau, which starts at increasingly smaller recrystallized fractions.

On the other hand, when precipitation starts, there is a considerable increase in the pinning forces and therefore a momentary inhibition of the recrystallization is

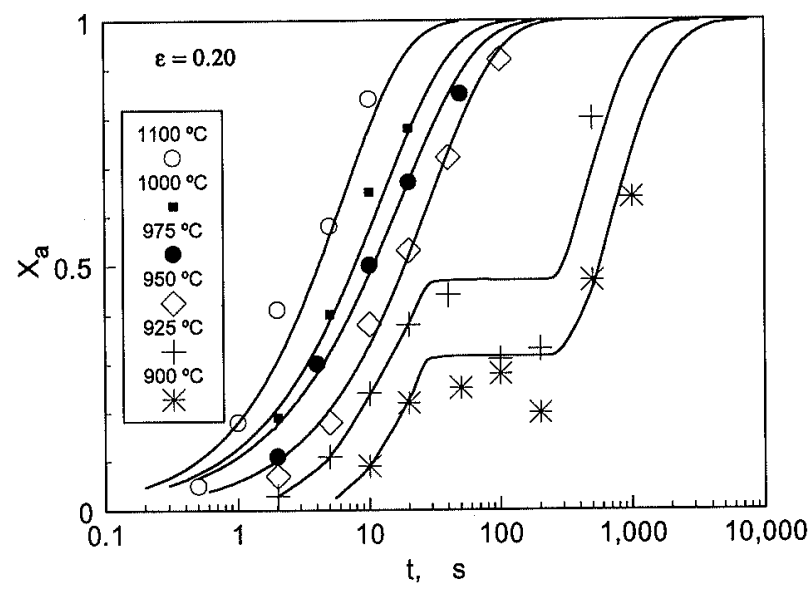

Fig. 1. Variation of the recrystallized fraction $\left(X_{\mathrm{a}}\right)$ with time ( $t$ ) for steel used. $\varepsilon=0.20 ; \dot{\varepsilon}=3.63 \mathrm{~s}^{-1}$. observed, which is manifested in the curves $X_{\mathbf{a}}(\operatorname{lnt})$ in the form of a plateau. Throughout the plateau there can also be a certain coalescence between precipitates, as has been observed by other authors, ${ }^{20)}$ though the heterogeneity in the sizes of the precipitates rather suggests that the precipitation continues throughout the plateau and that a part of the new precipitates do so on others already existing, in other words the first precipitates formed could serve as nuclei for others.

Nevertheless, in fact, the authors have seen by transmission electron microscopy (TEM) that the plateau starts when a certain fraction, which could be around $5 \%$, has already precipitated. The end of the plateau corresponds approximately to the end of precipitation, though the observations made to date do not permit us to establish with certainty the magnitude of this approximation. In this sense, a work related with precipitation with the assistance of (TEM) in $\mathrm{Nb}, \mathrm{V}$ and $\mathrm{Ti}$ microalloyed steels, is currently carrying out which will allow us to establish with greater exactitude the relative coincidence between the start and end of precipitation and of the plateau, respectively.

The curves corresponding to the strain of 0.35 are similar in form to the above (Fig. 2). For the same temperature, recrystallization is faster, i.e. the curves move towards the left (less time), and the tendency is noted of the appearance of the plateau at slightly lower temperatures.

It is deduced that induced precipitation is capable of momentarily blocking recrystallization. If advantage is taken of this time in rolling, or in any other hot forming process, it would be possible to obtain a highly deformed austenite $(\gamma)$ and consequently a very fine ferrite $(\alpha)$ after the $\gamma \rightarrow \alpha$ transformation.

PTT diagrams are easily drawn by simply noting on a system of time-temperature coordinates the start and finish times of each plateau, as well as the corresponding testing temperature. In this way, it is possible to draw the typical curves for the start $\left(P_{\mathrm{s}}\right)$ and finish $\left(P_{\mathrm{f}}\right)$ of induced precipitation. Furthermore, if in Figs. 1 and 2 we draw lines parallel to the time axis, at different recrystallized fractions, such as $0.1,0.3,0.5,0.9$ and 0.95 , and we note the temperatures and times which cor-

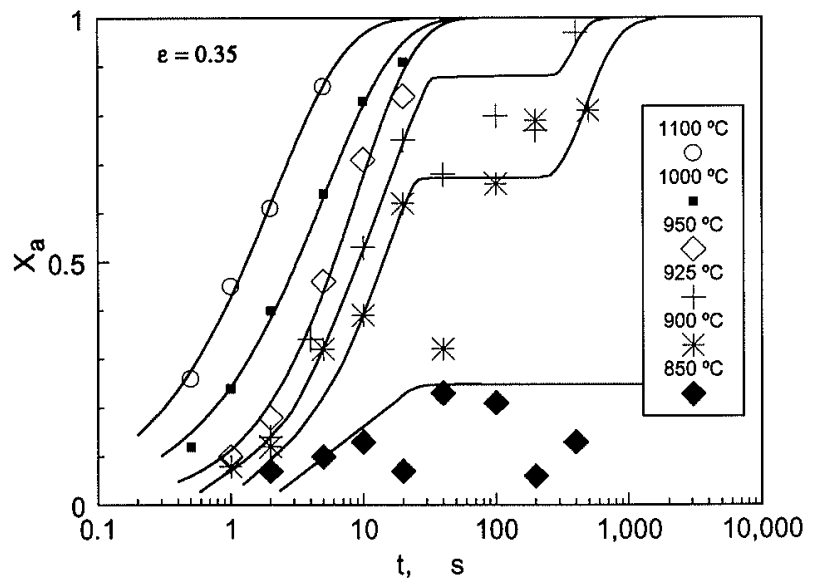

Fig. 2. Variation of the recrystallized fraction $\left(X_{\mathrm{a}}\right)$ with time ( $t$ ) for steel used, $\varepsilon=0.35 ; \dot{\varepsilon}=3.63 \mathrm{~s}^{-1}$. 
respond to the intersection points of these parallel lines with the recrystallization curves, and if these points are drawn on the PTT diagrams, the final result is Figs. 3 and 4, which correspond to the two strains 0.20 and 0.35 , respectively, known as recrystallization-precipitationtime-temperature (RPTT) diagrams. Each of these figures represents in graph form the $\mathrm{R}-\mathrm{P}$ interaction for the corresponding strain.

The RPTT diagrams obtained show clear differences in comparison with the hypothetical or theoretical diagrams proposed by other authors. ${ }^{19,20)}$ Firstly, in Figs. 3 and 4 it is seen that each line corresponding to a certain recrystallized fraction remains horizontal between the $P_{\mathrm{s}}$ and $P_{\mathrm{f}}$ curves, which is mathematically translated into a discontinuity of the function derived at the points of intersection with $P_{\mathrm{s}}$ and $P_{\mathrm{f}}$. This means that in order to obtain a certain recrystallized fraction at a temperature below the precipitation start temperature, it is necessary to take into account the duration time of the precipitation, as the recrystallization does not progress during this time. On the other hand, the nose of the curve $P_{\mathrm{s}}$ would intercept the line corresponding to a recrystallized fraction close to 0.5 . Furthermore, the lines corresponding to fractions equal to or less than 0.1 do not intercept the curve $P_{\mathrm{s}}$, in other words, precipitation only seems to occur if the recrystallized fraction is greater than 0.1 .

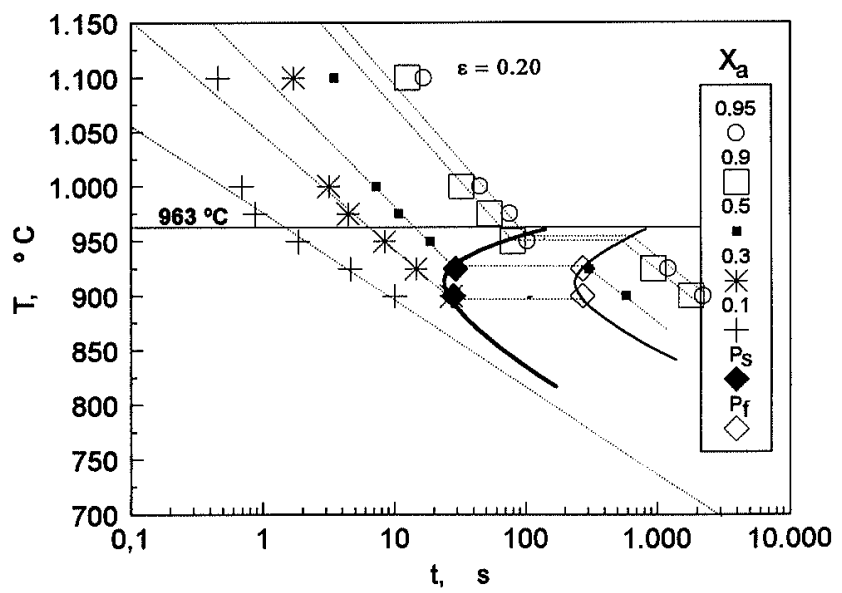

Fig. 3. RPTT diagrams and interaction recrystallization-precipitation. $\varepsilon=0.20$.

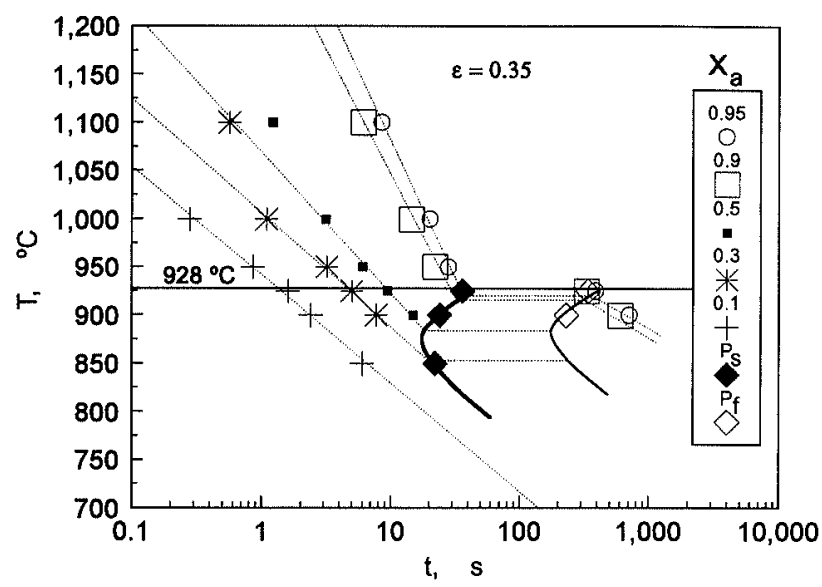

Fig. 4. RPTT diagrams and interaction recrystallization-precipitation. $\varepsilon=0.35$.
From the intersection of the different recrystallized fraction lines with the $P_{\mathrm{s}}$ curve, Fig. 5 is obtained, which represents the relation existing between the recrystallized fraction and the temperature at which induced precipitation starts. The intersection of the two regression lines with the horizontal line corresponding to a recrystallized fraction equal to 1 , makes it possible to determine SRCT, this being $1236 \mathrm{~K}\left(963^{\circ} \mathrm{C}\right)$ and $1201 \mathrm{~K}$ $\left(928^{\circ} \mathrm{C}\right)$ for the strains 0.20 and 0.35 , respectively. These values represent practically the asymptote for the $P_{\mathrm{s}}$ and $P_{f}$ curves, which has obviously facilitated their plotting (Figs. 3-4). In addition to SRCT, Table 2 contains the temperatures $\left(T_{\mathrm{N}}\right)$ and the minimum incubation times $\left(t_{\mathrm{N}}\right)$, corresponding to the nose of the $P_{\mathrm{s}}$ curves, and the solubility temperatures $\left(T_{\mathrm{s}}\right)$ deduced from the products of solubility. ${ }^{21)}$

It may be seen that at greater strains $(\varepsilon=0.35)$ the value of SRCT is lower. Comparison of SRCT values with the solubility temperatures calculated makes it possible to identify the nature of the precipitates. These are vanadium nitrides, as carbides are formed at lower temperatures. Furthermore, it is observed that SRCT is always lower than the solubility temperature, due to the fact that the strain induced precipitation moves away from the thermodynamic equilibrium conditions, with which the product of solubility are determined. An increase in the strain also reduces the nose temperature and the minimum incubation time. Furthermore, the interval during which precipitation takes place $\left(P_{\mathrm{f}}-P_{\mathrm{s}}\right)$ is smaller.

On the other hand, it should be noted that the minimum incubation time corresponds to recrystallized

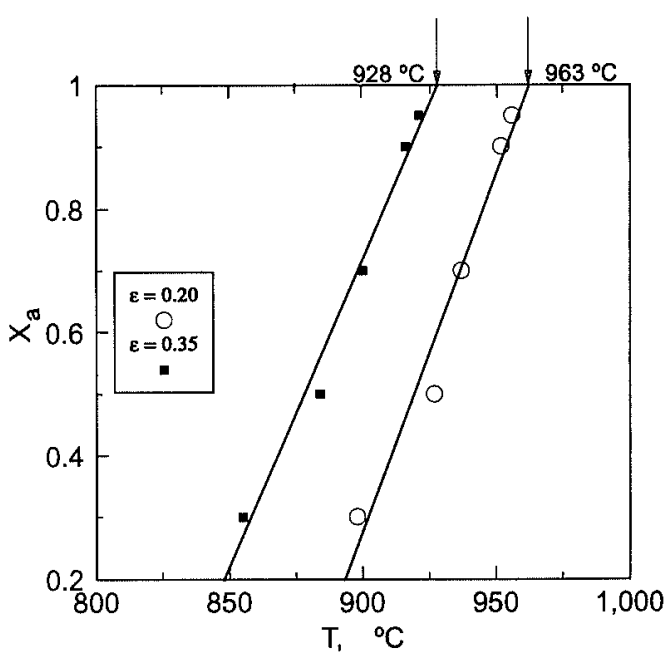

Fig. 5. Recrystallized fraction $\left(X_{\mathrm{a}}\right)$ against the temperature of intersection between the recrystallized fraction lines and $P_{\mathrm{s}}$ curve.

Table 2. Static recrystallization critical temperature (SRCT); nose temperature of $P_{\mathrm{s}}\left(T_{\mathrm{N}}\right)$; minimum incubation time $\left(t_{\mathrm{N}}\right)$ and solubility temperature $\left(T_{\mathrm{s}}\right)$.

\begin{tabular}{|c|c|c|c|c|}
\hline Strain & $\begin{array}{l}\text { SRCT, } \\
\mathrm{K}\left({ }^{\circ} \mathrm{C}\right)\end{array}$ & $\begin{array}{c}T_{\mathrm{N}}, \\
\mathrm{K}\left({ }^{\circ} \mathrm{C}\right)\end{array}$ & $\frac{t_{\mathrm{N}}}{\mathrm{s}}$ & $\begin{array}{c}T_{\mathrm{s}}, \\
\mathrm{K}\left({ }^{\circ} \mathrm{C}\right)\end{array}$ \\
\hline$\varepsilon=0.20$ & $1236(963)$ & $1188(915)$ & 24 & VN: I 365 (1 092) \\
\hline$\varepsilon=0.35$ & $1201(928)$ & $1151(878)$ & 18.5 & VC: $1177(904)$ \\
\hline
\end{tabular}


fractions close to 0.5 and that as the recrystallized fraction moves away from this value, either increasing or decreasing, the incubation time increases considerably. These results are similar to those found by other authors ${ }^{22)}$ and have served to reconsider the definition of the no-recrystallization temperature and to establish a new concept for this. ${ }^{23)}$

During the time in which precipitation takes place $\left(P_{\mathrm{f}}-P_{\mathrm{s}}\right)$ the recrystallized fraction can obviously not diminish, as the phenomenon is irreversible, even though in many cases the experimental points are situated below the corresponding curve $X_{\mathrm{a}}(t)$ (Figs. 1-2). The explanation for this is that the precipitation slightly hardens the austenite and the back extrapolation method which is used for calculating the recrystallized fraction is not capable of distinguishing between hardening by precipitation or hardening by the reduction in the recrystallized fraction. The constancy of the recrystallized fraction during precipitation is also translated into a horizontal line between the $P_{\mathrm{s}}$ and $P_{\mathrm{f}}$ curves, as shown in Figs. 3 and 4.

The kinetics of the static recrystallization of austenite can be described by an Avrami equation in the following way:

$$
X_{\mathrm{a}}=1-\exp \left[-0.693\left(\frac{t}{t_{0.5}}\right)^{n}\right]
$$

where $X_{\mathrm{a}}$ is the fraction of the recrystallized volume and $t_{0.5}$ is the time corresponding to half of the recrystallized volume, which depends practically on all the variables which intervene in hot deformation and whose most general expression follows a law of the type:

$$
t_{0.5}=A \varepsilon^{p} \dot{\varepsilon}^{q} D^{s} \exp \frac{Q}{R T}
$$

where $\varepsilon$ is the strain, $\dot{\varepsilon}$ the strain rate, $D$ the austenite grain size, $Q$ the activation energy, $T$ the absolute temperature and $R$ the gas constant.

After precipitation has finished, the different recrystallized fraction lines have a lower slope in absolute value, though they continue to be parallel as they were before precipitation (Figs. 3-4). In accordance with Eq. (2), the activation energy will be greater now than before the start of precipitation. The horizontal of $X_{0.5}$ between $P_{\mathrm{s}}$ and $P_{\mathrm{f}}$ gives the temperature at the moment when precipitation starts and finishes with a recrystallized fraction of $50 \%$. In the same way, the other horizontal lines drawn give the precipitation temperatures for the corresponding recrystallized fraction. If the logarithm of $t_{0.5}$ is represented against the inverse of the absolute temperature $(1 / T)$, for the two strains of 0.20 and 0.35 , we obtain the graph displayed in Fig. 6. In accordance with Eq. (2), the slope of each line multiplied by $R$ $(8.314 \mathrm{~J} \mathrm{~mol} / \mathrm{K})$ gives the value of $Q$ before and after precipitation. In the first stage, the value of $Q$ was $171000 \mathrm{~J} / \mathrm{mol}$. In the second stage, after precipitation, the activation energy was $397000 \mathrm{~J} / \mathrm{mol}$. In other words, the value of the activation energy changes from one phase to another, and this occurs at a constant temperature during the time that precipitation takes place. After precipitation, the activation energy has grown signifi-

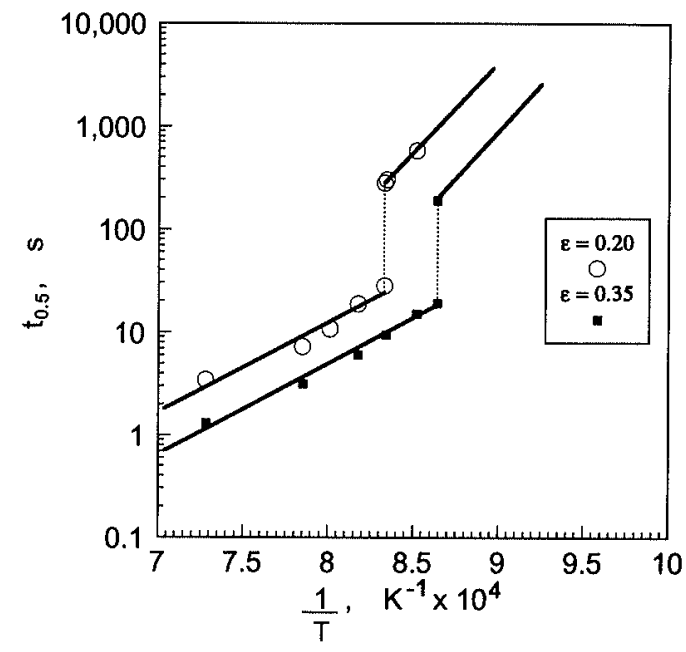

Fig. 6. Plot of $l_{0.5}$ against the reciprocal of the absolute temperature.

cantly, which is translated into greater difficulty for the austenite to recrystallize. Figure 6 represents a new concept of R-P interaction, which contrasts with other conceptions considered as classical ${ }^{24)}$ and represents an improvement on even other more recent ones. ${ }^{5)}$

Once precipitation has finished and though the precipitates are in general greater than at the start of precipitation, due to the coalescence between them and the fact that some have served as nucleation sites for others, the precipitates do not cease to exercise a certain pinning force on grain boundary migrating, which is translated into a greater difficulty for the progression of recrystallization, though logically the recrystallization driving forces are now greater than the pinning forces. Consequently, the activation energy is greater after precipitation than before.

On the other hand the value of $171000 \mathrm{~J} \mathrm{~mol}^{-1}$ obtained for the activation energy at temperatures above $\mathrm{SRCT}$, i.e. when the vanadium is in solution, coincides with the activation energy of other low carbon vanadium microalloyed steels. ${ }^{5)}$ This energy may also be calculated with the assistance of the recently published model. ${ }^{25}$ )

When deformation takes place in isothermal conditions, as in the torsion tests which were performed, precipitation only depends on time and it is not necessary to reduce the temperature for it to progress. Therefore, SRCT is defined as the temperature at which precipitation starts and finishes when the recrystallized fraction is $100 \%$. The activation energy increases discontinuously from temperatures above SRCT, where all the elements are in solution in the austenite, to temperatures below SRCT, where precipitation has already taken place. During hot rolling, though the plate or strip is continuously cooling, induced precipitation does not progress because of the effect of the cooling but because of the time which passes between successive passes. When the times between passes and the temperatures are close to those of the nose of the $P_{\mathrm{s}}$ curve, precipitation will start and will be completed more quickly, but the recrystallized fraction will not be $100 \%$ but much lower, in the region of $50 \%$. A parallel study, consisting of torsion simulations of 18 rolling passes in an $\mathrm{Nb}$ microalloyed steel, was 
carried out to determine $T_{\mathrm{nr}}$, reaching the conclusion that the microstructure immediately before reaching $T_{\mathrm{nr}}$ contained a recrystallized fraction of approximately $50 \%$ when the interpass time was to the order of the minimum incubation time of the precipitates $\left(t_{\mathrm{N}}\right){ }^{23}$ )

Some microstructures were determined with the aim of showing visually the phenomenon of recrystallization inhibition due to precipitation. First of all, Fig. 7 shows the reference microstructure, corresponding to the reheating temperature $\left(1200^{\circ} \mathrm{C} \times 10 \mathrm{~min}\right)$, where the austenite grain size obtained can be seen, whose average size, as mentioned above, was $157 \mu \mathrm{m}$. The three following microstructures were obtained taking into account the $900^{\circ} \mathrm{C}$ curve in Fig. 2 and selecting the following times after applying the strain of 0.35 : $7 \mathrm{~s}$ (Fig. 8); $40 \mathrm{~s}$ (Fig. 9) and $250 \mathrm{~s}$ (Fig. 10). The times chosen correspond, as can be seen, to a point in time before the start of the plateau, once the plateau has started and at the end of plateau. At the end of each time the specimen was quenched and the microstructure was observed on a longitudinal surface close to the cylindrical surface of the sample, exactly at $0.35 \mathrm{~mm}$ from the surface, in other words $2.65 \mathrm{~mm}$ from the axis. The recrystallized

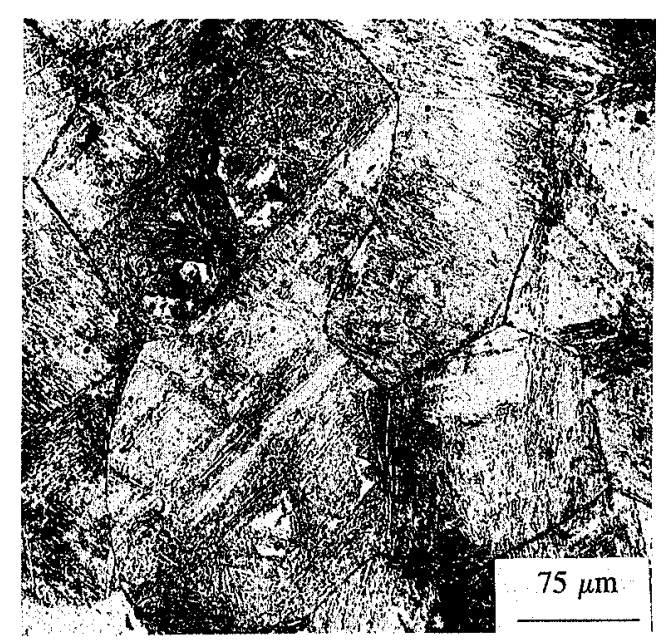

Fig. 7. Microstructure of austenite grain at reheating temperature $\left(1200^{\circ} \mathrm{C} \times 10 \mathrm{~min}\right)$.

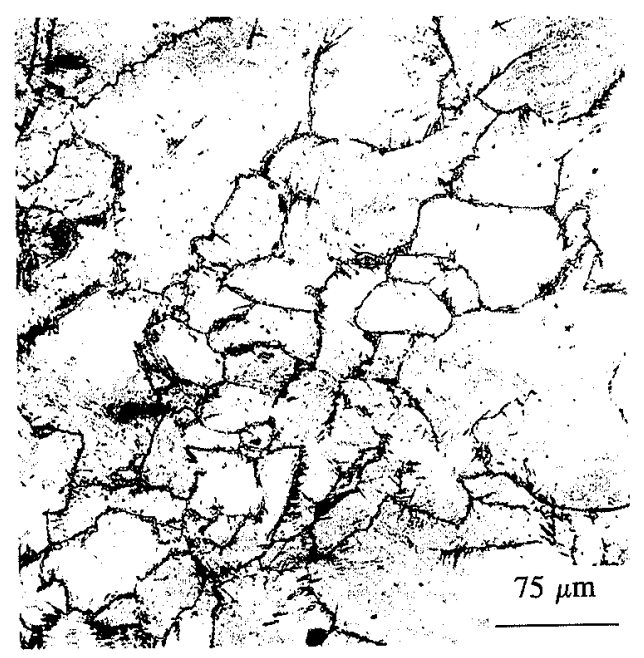

Fig. 8. Microstructure of recrystallized fraction in austenite deformed at $900^{\circ} \mathrm{C}, \varepsilon=0.35$, and holding time $7 \mathrm{~s}$. $\left(X_{\mathrm{a}}=0.35\right)$.

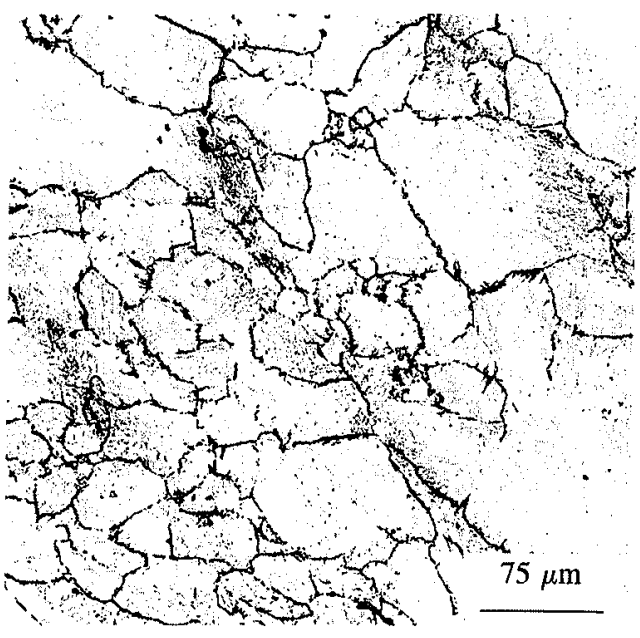

Fig. 9. Microstructure of recrystallized fraction in austenite deformed at $900^{\circ} \mathrm{C}, \varepsilon=0.35$, and holding time $40 \mathrm{~s}$ $\left(X_{\mathrm{a}}=0.60\right)$.

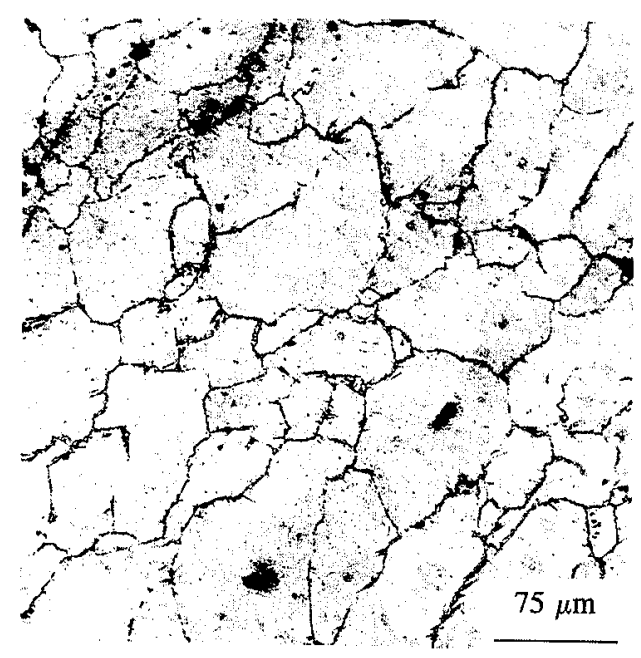

Fig. 10. Microstructure of recrystallized fraction in austenite deformed at $900^{\circ} \mathrm{C}, \varepsilon=0.35$, and holding time $250 \mathrm{~s}$. $\left(X_{\mathrm{a}}=0.70\right)$

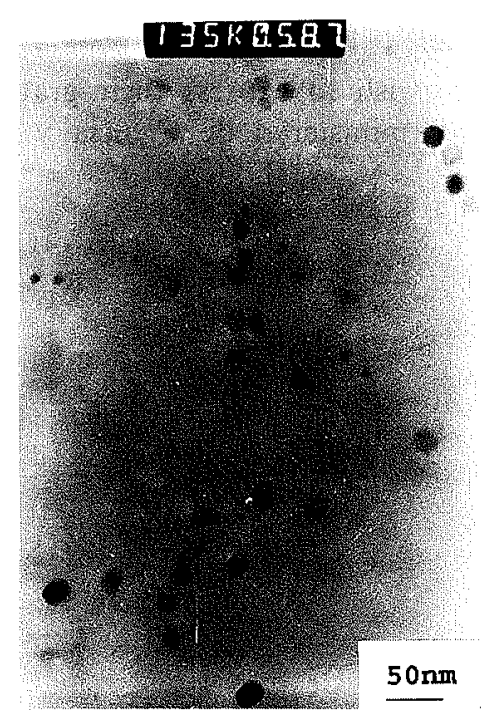

Fig. 11. Carbon extraction replicas showing the precipitates of vanadium: $\varepsilon=0.35 ; \dot{\varepsilon}=3.63 \mathrm{~s}^{-1}$; test temperature $=$ $900^{\circ} \mathrm{C} ;$ time after strain $=40 \mathrm{~s}$. 
fraction was measured by optical microscope observation of more than 10 fields and has been noted at the foot of the figures. Its comparison with the recrystallized fraction determined by the back extrapolation method indicates that this method has acceptable precision.

Finally, Fig. 11 shows the vanadium nitride precipitates observed on the sample in Fig. 9, whose strain conditions are shown at the foot of the figure. An ultrathin sample was taken in the longitudinal direction, situated at $2.65 \mathrm{~mm}$ from the axis of the sample, and was observed TEM using the carbon extraction replicas technique. The spectrum of the analysis showed the relatively high vanadium content and the presence of nitrogen. The distribution of the precipitates was rather heterogenous and the size of the particles oscillated between 10 and $25 \mathrm{~nm}$, whose weighted mean value was approximately $16 \mathrm{~nm}$.

\section{Conclusions}

(1) The methodology applied makes it possible to determine RPTT diagrams and recrystallization-precipitation interaction.

(2) At greater strains, the times for the incubation of the precipitates and for complete precipitation are smaller.

(3) The start of recrystallization-precipitation interaction is at the "nose" of the $P_{\mathrm{s}}$ curve when the recrystallized fraction is approximately equal to 0.5 . When the fraction is less than 0.5 , the incubation time becomes increasingly longer and when the fraction reaches even lower values $(<0.2)$ nucleation of the precipitates is practically impossible.

(4) Activation energy changes discontinuously at SRCT, being much greater after precipitation.

(5) Precipitation is possible for any recrystallized fraction upper to 0.1 , depending on the holding time at the corresponding temperature.

(6) In hot rolling with smaller interpass times, precipitation would start when the recrystallized fraction is close to 0.5 .

\section{Acknowledgements}

The authors wish to express their gratitude to the "Comisión Interministerial de Ciencia y Tecnología"
(CICYT) for the financing granted to project MAT 94-0798, within the framework of which this work has been carried out, and to the Agencia Española de Cooperación Internacional (AECI) through the "Instituto de Cooperación Iberoamericana" (ICI) for financing the stay of Eng. A. Quispe, in CENIM.

\section{REFERENCES}

1) F. H. Samuel, S. Yue, J. J. Jonas and B. A. Zbinden: ISIJ Int., 29 (1989), 878

2) S. F. Medina and J. E. Mancilla: Acta Metall. Mater, 42 (1994), 3945.

3) S. F. Medina and J. E. Mancilla: Scr. Metall. Mater., 31 (1994), 315.

4) S. F. Medina and J. E. Mancilla: Scr. Metall. Mater, 32 (1995), 43.

5) S. F. Medina, J. E. Mancilla and C. A. Hernández: ISIJ Int., 34 (1994), 689

6) S. F. Medina and J. E. Mancilla: Scr. Metall. Mater, 30 (1994), 73.

7) S. F. Medina and J. E. Mancilla: ISIJ Int., 33 (1993), 1257.

8) B. Dutta and C. M. Sellars: Mater. Sci. Technol., 3 (1987), 197.

9) S. Okaguchi and T. Hashimoto: ISIJ Int., 32 (1992), 283.

10) S. H. Park, S. Yue and J. J. Jonas: Metall. Trans., 23A (1992), I64I.

11) D. Q. Bai, S. Yue, W. P. Sun and J. J. Jonas: Metall. Trans., 24A (1993), 2151.

12) W. P. Sun, M. Militzer, D. Q. Bai and J. J. Jonas: Acta Metall. Mater., 41 (1993), 3595.

13) A. Le Bon, J. Rofes-Vernis and C. Rossard: Met. Sci., 9 (1975), 36.

14) W. J. Liu and J. J. Jonas: Metall. Trans., 19A (1988), 1403.

15) W. J. Liu and J. J. Jonas: Metall. Trans., 20A (1989), 689.

16) A. Faessel: Rev. Metall. Cah. Inf. Tech., 33 (1976), 875.

17) H. L. Andrade, M. G. Akben and J. J. Jonas: Metall. Trans., 14A (1983), 1967.

18) P. Choquet, A. Le Bon and C. Perdrix: Proc. Int. Conf. on Strength of Metals and Alloys, CISMA 7, Vol. 3, ed. by H. J. Mcqueen, J. B. Bailon, J. I. Dickson, J. J. Jonas and M. G. Akben, Pergamon Press, Oxford, (1986), 1205.

19) S. S. Hansen, J. B. Bander and M. Cohen: Metall. Trans., 11A (1980), 387.

20) O. Kwon and J. DeArdo: Acta Metall. Mater., 39 (1990), 529.

21) K. Narita: Trans. Iron Steel Inst. Jpn., 15 (1975), 145.

22) D. Q. Bai, S. Yue, W. P. Sun and J. J. Jonas: Metall. Trans., 24A (1993), 2151.

23) S. F. Medina: Mater. Sci. Technol., accepted to be published.

24) C. M. Sellars: Proc. Int. Conf. on Hot Working and Forming Processes, ed. by C. M. Sellars and G. J. Davies, Met. Soc., London, (1980), 3.

25) S. F. Medina and J. E. Mancilla: ISIJ Int., 36 (1996), 1070. 